\title{
Die digitale Intensivstation
}

\section{Emanuela Keller}

Leitende Ärztin der Neurochirurgischen Intensivstation am UniversitätsSpital Zürich

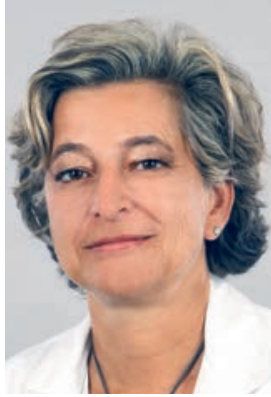

Die Digitalisierung ist in aller Munde: Wie verändert sie das Gesundheitswesen? Welchen Nutzen hat sie für die Patientinnen und Patienten? Wird sie helfen, Kosten zu sparen, oder wird sie im Gegenteil ein weiterer Kostentreiber? Ich möchte diesen Fragen am Beispiel einer Intensivstation nachgehen. Die Intensivmedizin ist bereits seit den 1990er Jahren digitalisiert. Damals kamen die ersten Geräte auf, die Daten nicht mehr analog, sondern digital verarbeiten, anzeigen und speichern konnten. In den vergangenen zehn Jahren kam vor allem die digitale Dokumentation der Krankengeschichten hinzu. Intensivstationen waren vermutlich der erste Bereich der Medizin, in dem Big Data zum Thema wurde. Die diversen Geräte auf unserer Station erzeugen pro Tag und Patient bis zu 60 Terabyte an Daten.

\section{Intensivstationen waren vermutlich der erste Bereich der Medizin, in dem Big Data zum Thema wurde.}

Künstliche Intelligenz kann uns helfen, die relevanten Informationen herausfiltern. Zuvor gilt es aber ein Problem zu lösen. Die heutigen Geräte exportieren ihre Daten in unterschiedlichen Formaten und können nicht miteinander kommunizieren. Die Beatmungsmaschine kennt die Herzfrequenz des Patienten nicht, und das EKG-Gerät weiss nichts über den Sauerstoffgehalt im Blut. Entsprechend können diese Werte nicht miteinander in Bezug gesetzt werden. Eine wirkliche Digitalisierung im Gesundheitswesen erreichen wir so nicht. Die Daten der verschiedenen Geräte müssen integriert werden.

Man kann es vergleichen mit den verschiedenen Verfahren der Bildgebung. Dort dauerte es rund 20 Jahre, bis sich die Gerätehersteller auf einen gemeinsamen Datenstandard einigten. Seit knapp 10 Jahren existiert ein solcher, was ein enormer Vorteil ist beim Austausch von Daten aus der Bildgebung. Dasselbe müssen wir auch für medizintechnische Geräte erreichen. Ich bin überzeugt, dass das kommen wird. Die Gerätehersteller haben ein Interesse daran, sich damit einen Wettbewerbsvorteil zu verschaffen. Aber bis es so weit ist, suchen wir Universitätsspitäler bereits nach entsprechenden Lösungen. Im Rahmen des Projektes «ICU Cockpit»* arbeiten wir auf der Neurochirurgischen Intensivstation am UniversitätsSpital Zürich daran, die Geräte zu integrieren und künstliche Intelligenz nutzbar zu machen. Dies unter anderem, um voraussagen zu können, wie sich der Gesundheitszustand eines Patienten entwickeln wird. Zudem soll uns die künstliche Intelligenz therapeutische Empfehlungen geben. Erste Anwendungen davon funktionieren im Alltag bereits. Selbstverständlich werden die Daten der Patientinnen und Patienten dabei immer in anonymisierter Form genutzt.

Ich bin überzeugt: Die Digitalisierung hilft uns, die Medizin sicherer zu machen. Mehr Sicherheit erreicht man, indem die Menschen von stereotypen, ermüdenden Arbeiten entlastet werden. So haben sie den Kopf frei für den Kontakt mit den Patientinnen und Patienten sowie Angehörigen oder können sich drängenden ethischen Fragen widmen. Insofern hilft die Digitalisierung auch, die Intensivstation humaner zu gestalten. Und sie wird helfen, die Kosten zu senken - Stichwort Telemedizin. Patientinnen und Patienten werden in Zukunft früher von der Intensivstation auf eine normale Pflegestation wechseln oder gar nach Hause gehen - dank dem Monitoring durch Biosensoren, die sie am Körper tragen. Gekoppelt mit Algorithmen werden die Sensoren einen bedrohlichen Zustand vorhersagen, und wir werden entsprechend früh genug reagieren können.

Auch ausserhalb von Intensivstationen sehe ich für die Digitalisierung ein grosses Potenzial. Sie wird sowohl den Menschen nützen wie auch Kosten sparen helfen. Dazu müssen wir die Prävention stärken, also verhindern, dass Menschen überhaupt in lebensbedrohliche Situationen geraten. Dank Telemedizin können wir intervenieren, bevor es dazu kommt. Gerade für Länder mit weniger Ressourcen ist das eine grosse Chance. In Afrika oder Indien beispielsweise gibt es ländliche Gegenden fast ohne Gesundheitsversorgung. Da könnte man mit Apps und Telemedizin die Gesundheit von Millionen von Menschen mit einfachen Massnahmen verbessern. Nutzen wir diese Chancen! 